\title{
Innovative Method Using Adhesive Force for Surface Micromachining of Carbon Nanowall
}

\author{
Hyeokjoo Choi ${ }^{1}{ }^{\mathbb{D}}$, Seokhun Kwon ${ }^{1}$, Seokwon Lee ${ }^{1}$, Yonghyeon Kim ${ }^{2}$, Hyunil Kang ${ }^{1}$, \\ Jung Hyun Kim ${ }^{2}$ (i) and Wonseok Choi ${ }^{1, *(D)}$ \\ 1 Department of Electrical Engineering, Hanbat National University, Daejeon 34158, Korea; \\ hyukju1210@hanmail.net (H.C.); kwon1567@naver.com (S.K.); dltjrdnjs000@naver.com (S.L.); \\ hikang@hanbat.ac.kr (H.K.) \\ 2 Department of Advanced Materials Engineering, Hanbat National University, Daejeon 34158, Korea; \\ kjw4493@naver.com (Y.K.); jhkim2011@hanbat.ac.kr (J.H.K.) \\ * Correspondence: wschoi@hanbat.ac.kr
}

Received: 10 August 2020; Accepted: 28 September 2020; Published: 6 October 2020

\begin{abstract}
The application of a carbon nanowall (CNW) via transfer is very demanding due to the unusual structure of vertically grown wall-shaped that easily collapses. In addition, direct growth on a device cannot obtain a precision-patterned shape because of the temperature limit of the photoresist (PR). Therefore, in this paper, we demonstrate a new CNW surface micromachining technology capable of direct growth. In order to reduce unexpected damage caused by chemical etching, a physical force was used to etch with the adhesive properties of CNWs that have low adhesion to silicon wafer. To prevent compositing with PR, the CNW was surface modified using oxygen plasma. Since there is a risk of surface-modified CNW (SMCNW) collapse in an ultrasonic treatment, which is a physical force, the CNW was coated with PR. After etching the SMCNW grown on PR uncoated area, PR was lifted off using an acetone solution. The effect on the SMCNW by the lift-off process was investigated. The surface, chemical, and structural properties of PR-removed SMCNW and pristine-SMCNW were compared and showed a minimal difference. Therefore, the CNW surface micromachining technique was considered successful.
\end{abstract}

Keywords: carbon nanowall; sonication; surface micromachining; surface modification

\section{Introduction}

Graphite-related materials, such as graphene, carbon nanotubes (CNTs), and carbon nanowalls (CNWs) have continuously attracted interest in many fields [1-10]. A wall-shaped CNW is based on graphene sheets standing vertically, to which electrons or desired materials can attach to on both sides because CNWs have a lamella structure and the largest reaction area among these materials. Furthermore, CNW, unlike graphene and CNTs, does not require a metal catalyst, such as copper [11], nickel [12], gold [13], or platinum [14], during the growth process. At a relatively low temperature, compared to other graphite-related materials, a catalyst-free $\mathrm{CNW}$ is grown using various chemical vapor deposition (CVD) techniques or a sputtering system [15-19].

The CVD method offers easy to synthesize thin-films, such as silicon-based $\left(\mathrm{SiO}_{2}, \mathrm{SiN}_{x}, \mathrm{SiO}_{x} \mathrm{~N}_{y}\right.$, a-Si, etc.) and carbon thin-films with high purity materials that have good stability and uniformity. In the synthesis mechanism of CNW via CVD, carbon radicals separate from the injected source gas in the chamber and are adsorbed on the surface of the substrate to create several layers of graphene, and the wall grows vertically at the graphene's defect site [20,21]. In the case of branches that grow beside the nanowalls of $\mathrm{CNW}$, they grow according to subtle variations in temperature and source power [22,23]. 
With regard to the growth of graphite-related materials through the CVD method, which requires a high temperature, directly growing on a desired area in the photolithography process from the photoresist (PR) temperature poses a limitation. Therefore, graphite-related materials have been grown on other substrates and then transferred to the wanted layer via microfabrication [24]. Since wall-shaped CNWs have a risk of collapse, its transfer is very demanding and, to the best of the authors' knowledge, very little is known about CNW micromachining techniques. Accordingly, there are limitations in terms of application and requires further studies because CNWs cannot selectively grow in the patterned area with precision.

In this work, surface micromachining of a CNW through surface modification and physical force without chemical etching is suggested. A CNW was grown on the substrate via microwave-plasma-enhanced CVD (MPECVD) with a mixture of methane $\left(\mathrm{CH}_{4}\right)$ and argon (Ar) gases at high temperatures $\left(600^{\circ} \mathrm{C}\right)$. When a non-polar $\mathrm{CNW}$ is coated with a non-polar PR, the two are very strongly attracted to each other, and the PR is not completely removed in the development process after exposure. To resolve this problem, the $\mathrm{CNW}$ was surface modified under oxygen $\left(\mathrm{O}_{2}\right)$ plasma to lower the strong adhesion between the PR and the CNW by a surface polarity change. The required area was coated with the $\mathrm{PR}$, and the undesired region was removed through sonication in deionized (DI) water. After removing the PR using acetone, the surface, structural, and chemical changes of the surface-modified CNWs (SMCNW) were observed. It was confirmed whether the CNW remained in the area that had been removed through sonication.

\section{Materials and Methods}

\subsection{Reagents and Materials}

Silicon (Si) wafers (100, p-type) were purchased from Taewon Scientific Co., Ltd. (iTASCO, Seoul, Korea). Trichloroethylene $(\geq 99.5 \%)$, acetone $(\geq 99.5 \%)$, and methanol ( $\geq 99.8 \%)$ solutions were purchased from Sigma-Aldrich. DI water $\left(\geq 18.2 \mathrm{M} \Omega \cdot \mathrm{cm} @ 25^{\circ} \mathrm{C}\right)$ was obtained using a water purification system (YOUNG IN Chromass Co., Ltd., Anyang-si, Korea, aquapuri 5 series). Silicon dioxide $\left(\mathrm{SiO}_{2}, 99.999 \%\right)$ and silver $(\mathrm{Ag}, 99.99 \%)$ targets were purchased from Taewon Scientific Co., Ltd. (iTASCO, Seoul, Korea). PR (AZ GXR-601 46cp) and its developer (AZ $300 \mathrm{MIF}$ ) were purchased from Sigma-Aldrich (St. Louis, MO, USA).

\subsection{Preparing the Substrates and Deposition of Adhesion-Increased Layer}

To clean the surface of a 4-inch Si wafer, which was the starting material, ultrasonic cleaning was performed using trichloroethylene, acetone, methanol, and DI water, in that order, for $10 \mathrm{~min}$ each. The $\mathrm{SiO}_{2}$ and $\mathrm{Ag}$ layers were deposited using a radio frequency (RF) magnetron sputtering system under different conditions using their 4-inch targets (Table 1). In the patterned area where the CNW should have remained, $\mathrm{a} \mathrm{SiO}_{2}$ layer, which was used as an insulator, was first deposited, followed by Ag. The Ag layer, a transition metal, was used to improve the adhesion between the CNW and its under layer.

Table 1. Radio frequency (RF) magnetron sputtering condition of $\mathrm{SiO}_{2}$ and $\mathrm{Ag}$ target.

\begin{tabular}{ccc}
\hline Parameter & $\mathrm{SiO}_{2}$ Target & Ag Target \\
\hline Substrate temperature & Room temperature & Room temperature \\
Injection gas & $\mathrm{Ar}: 34 \mathrm{sccm}$ & $\mathrm{Ar}: 40 \mathrm{sccm}$ \\
RF power & $\mathrm{O}_{2}: 6 \mathrm{sccm}$ & $150 \mathrm{~W}$ \\
Deposition time & $150 \mathrm{~W}$ & $3 \mathrm{~min}$ \\
Working pressure & $60 \mathrm{~min}$ & $1.5 \times 10^{-2} \mathrm{Torr}$ \\
\hline
\end{tabular}




\subsection{Growth of CNW and Its Surface Modification}

After the prepared Si wafer was placed on the stage inside the MPECVD (ASTeX-type, Woosin CryoVac, Uiwang-si, Korea, $2.45 \mathrm{GHz}$ microwave) chamber, a $10^{-4}$ Torr base vacuum was maintained. To form a plasma ball on the substrate producing $1300 \mathrm{~W}$ of microwave power, $20 \mathrm{sccm} \mathrm{CH}_{4}$ and Ar, respectively, were injected into the chamber. The CNW was subsequently grown on the entire substrate for $10 \mathrm{~min}$ in a working vacuum of $10^{-2} \mathrm{Torr}$ and at a $600{ }^{\circ} \mathrm{C}$ stage temperature. After $\mathrm{CNW}$ growth completion, the CNW was surface modified via plasma treatment, by injecting $20 \mathrm{sccm}$ of $\mathrm{O}_{2}$ gas with $200 \mathrm{~W}$ RF power for $2 \mathrm{~min}$. The SMCNW was etched with the nanowall branches and edge parts, and was polarized through attachment of the functional group.

\subsection{Innovative Method of Top-Down Surface Micromachining of CNW Based on its Adhesive Properties}

Carbon etching techniques using oxidation have been extensively studied [25,26]. If the metal used as an electrode, however, is unintentionally oxidized in the application, such as a sensor, the energy band gap of the metal electrode employed by the metal oxide layer may increase, causing the sensor's sensitivity to decrease. Therefore, an etching technique using physical force that does not employ a chemical etchant and using the CNW's adhesive properties was devised. A PR layer was used for coating the sample on which the SMCNW was grown. The PR we applied was a mixture of diazonaphthoquinone sulfonic ester and cresol novolak resin. Therefore, it can be inferred that the PR we used is a $p$-type that dissolves easily in a base due to its solubility variation in the UV absorbed part. The PR was coated on a flat material and it was spin-coated to have a height of about $3 \mu \mathrm{m}$. After the PR-coated sample was exposed to a $180 \mathrm{~mJ}$ UV light as part of the development process, the exposed PR area was dissolved using its developer. Because the adhesion between the CNW and the Si wafer was feeble, more than half of the undesired area of the CNW was dissolved along with the PR during the development process. The remaining $\mathrm{CNW}$ that needed to be removed was placed in DI water and was sonicated for $10 \mathrm{~min}$. To prevent deformation of the PR, the SMCNW was etched in minimal light. The CNWs in all the unwanted areas that had been etched were immersed in an acetone solution to remove the PR. After the PRs were completely removed, the CNWs were soaked in DI water and were immediately taken out and then dried naturally at room temperature. Figure 1 shows the overall fabrication process of SMCNW surface micromachining.

\subsection{Characterization and Measurement of the Samples}

By confirming the $\mathrm{C}$ atoms by energy-dispersive $\mathrm{X}$-ray spectroscopy (EDS) analysis, it was demonstrated whether the residual of the SMCNW was etched completely by the physical force. The contact angle, which was averaged for $5 \mathrm{~s}$ immediately after the drop, was observed by dropping $3 \mu \mathrm{L}$ DI water, a polar molecule, on the sample to check whether the CNW and SMCNW were polar or non-polar. The differences before and after surface micromachining of the SMCNW grown in the desired area were compared in terms of their surface, structural, and chemical properties. The surface characteristics were determined via field emission scanning electron microscopy (FESEM, HITACHI, Tokyo, Japan, S-4800) images and atomic force microscopy (AFM, Suwon-si, Korea, Park systems, XE-100; scanning size: $2 \mu \mathrm{m} \times 2 \mu \mathrm{m}$ ). Raman spectroscopy (NOST, Seongnam-si, Korea, FEX; excitation wavelength: $\sim 531 \mathrm{~nm}$; excitation power: $\sim 0.3 \mathrm{~mW}$ ) was conducted to observe the structural characteristics of the samples. From the Raman spectrum, inherent peaks of the graphite-related materials, and assorted information, such as the defects, the thickness of the graphene layers, and the distinctive structural properties of the $\mathrm{CNW}$, were obtained. The chemical properties of the samples were investigated using X-ray photoelectron spectroscopy (XPS, ThermoFisher Scientific, Waltham, MA, USA, K-Alpha+) analysis, which showed the chemical composition and the 1-10 nm bonding thickness of the surface. 
(a)

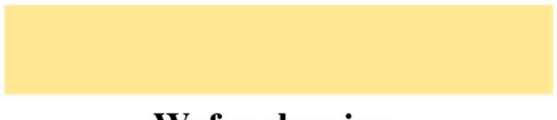

Wafer cleaning

(b)

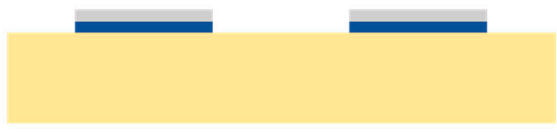

Interlayer deposition

(c)

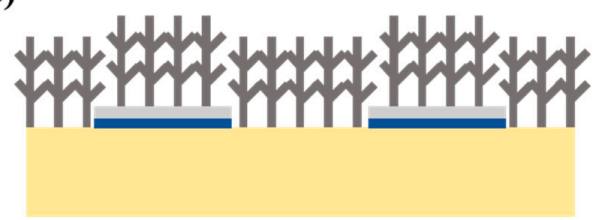

Growth of CNW

(d)

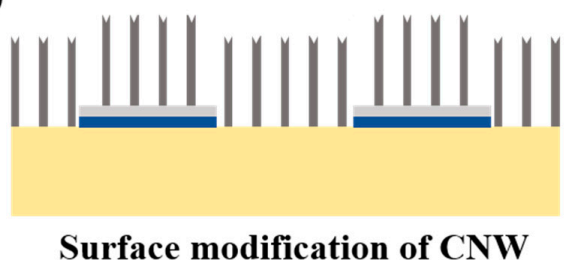

(e)

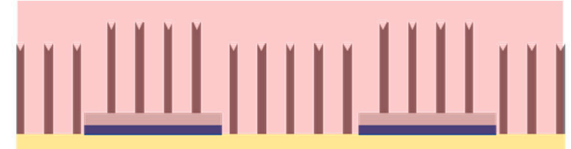

PR layer coating

(f)

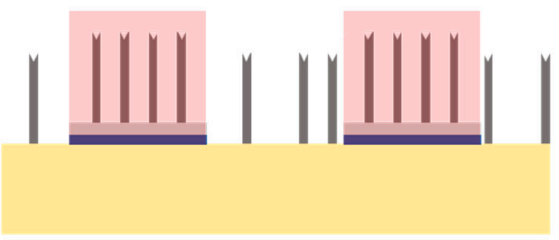

Exposure and develop

(g)

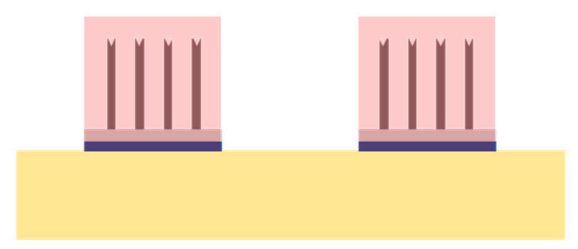

CNW etched by sonication

(h)

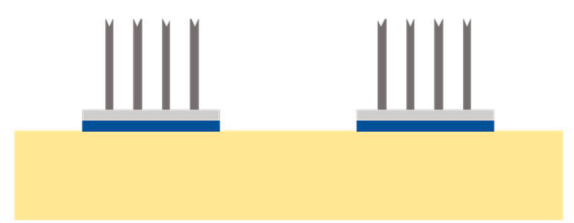

PR lift off

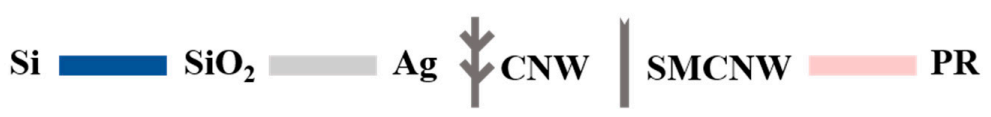

Figure 1. Fabrication process of top-down surface micromachining in surface-modified carbon nanowall. (a) Wafer cleaning process; (b) Patterning and deposition of $\mathrm{Ag} / \mathrm{SiO}_{2}$ layer; (c) Growth of $\mathrm{CNW}$ via MPECVD; (d) Surface modification of $\mathrm{CNW}$ by $\mathrm{O}_{2}$ plasma; (e) Coating the PR layer on the sample; (f) Exposure and develop process; (g) Surface micromachining of SMCNW by sonication; (h) PR lift-off process.

\section{Results and Discussion}

\subsection{Application of the CNW Photolithography Process}

Figure 2 shows the surface morphology of $\mathrm{SiO}_{2}$ and $\mathrm{Ag} / \mathrm{SiO}_{2}$ deposited on the cleaned $\mathrm{Si}$ wafer. Figure 2a,b shows the thickness of the $\mathrm{SiO}_{2}$ layer and the Ag layer, respectively, with the $\mathrm{Ag} / \mathrm{SiO}_{2}$ layer being about $180 \mathrm{~nm}$ thick. In Figure 2c, the average roughness (Ra) of the $\mathrm{SiO}_{2}$ layer is $0.63 \mathrm{~nm}$. The skewness of the $\mathrm{SiO}_{2}$ layer is -0.473 , which is a negative value, so it has an upwardly skewed roughness. Figure $2 \mathrm{~d}$ shows the surface roughness of the $\mathrm{Ag} / \mathrm{SiO}_{2}$ layer, while $\mathrm{Ra}$ is 4.788 , which has a rougher surface than the $\mathrm{SiO}_{2}$ layer. The skewness of $\mathrm{Ag} / \mathrm{SiO}_{2}$ is -1.333 , which has an upwardly skewed roughness. 

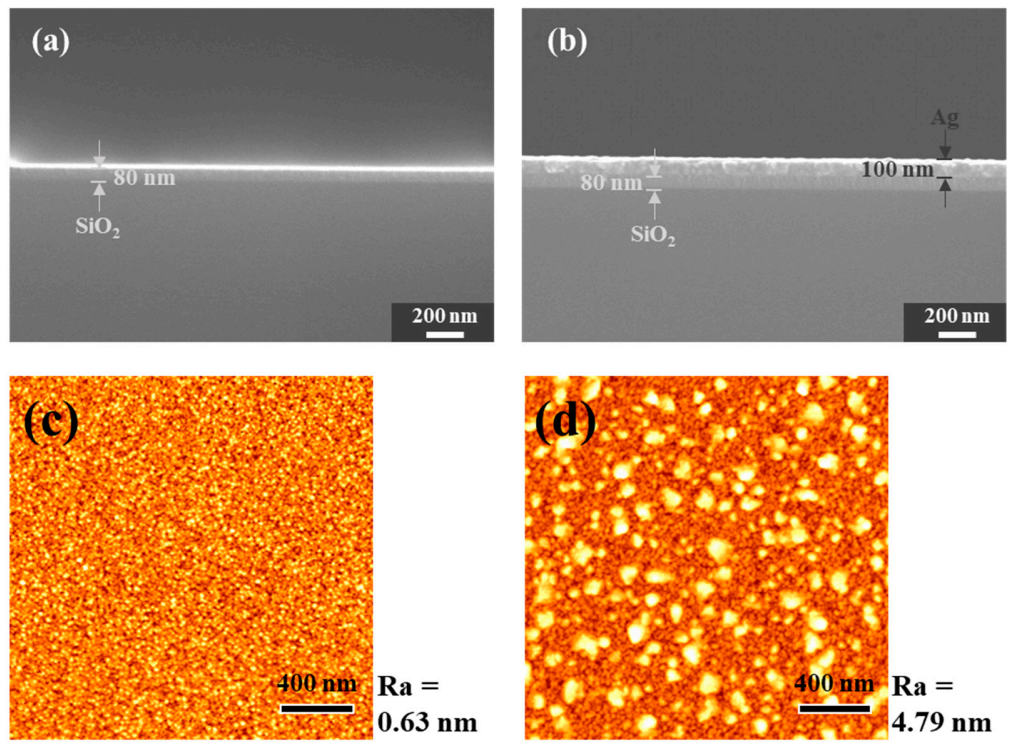

Figure 2. FESEM cross-section image of (a) $\mathrm{SiO}_{2}$ and (b) $\mathrm{Ag} / \mathrm{SiO}_{2}$ deposited $\mathrm{Si}$ wafer; atomic force microscopy (AFM) 2D image of (c) $\mathrm{SiO}_{2}$ and (d) $\mathrm{Ag} / \mathrm{SiO}_{2}$ layer.

Positive PR was patterned for CNW surface micromachining. Figure 3a shows a FESEM cross-sectional image of the $\mathrm{CNW}$ grown on a $\mathrm{Ag} / \mathrm{SiO}_{2}$ layer. The height of the $\mathrm{CNW}$ grown on the $\mathrm{Ag} / \mathrm{SiO}_{2}$ layer was about $1.7 \mu \mathrm{m}$, and the height of the CNW composited with a PR (PR@CNW) in Figure $3 \mathrm{~b}$ was about $1.9 \mu \mathrm{m}$. PR@CNW material was obtained by coating PR on CNW without surface modification, exposing it to UV light, and dissolving PR in a developer. However, during the development process, the PR area exposed to light was hardly dissolved in the developer and was not removed easily, even after sonicating in DI water. Through the comparison of the PR@CNW and CNW images, the CNW and PR were bonded with strong adhesion even after the development process. Figure $3 c$ confirms that the unexposed area retained its pyramid shape due to the characteristics of the positive PR. In addition, as shown in Figure 3d, even when the wall-shaped CNW was coated with a $\mathrm{PR}$, it was uniformly applied with a thickness of about $4 \mu \mathrm{m}$. It was spin-coated in a flat material so that the PR was about $3 \mu \mathrm{m}$; however, when PR was coated onto CNW, PR@CNW was formed and the height was $4 \mu \mathrm{m}$.
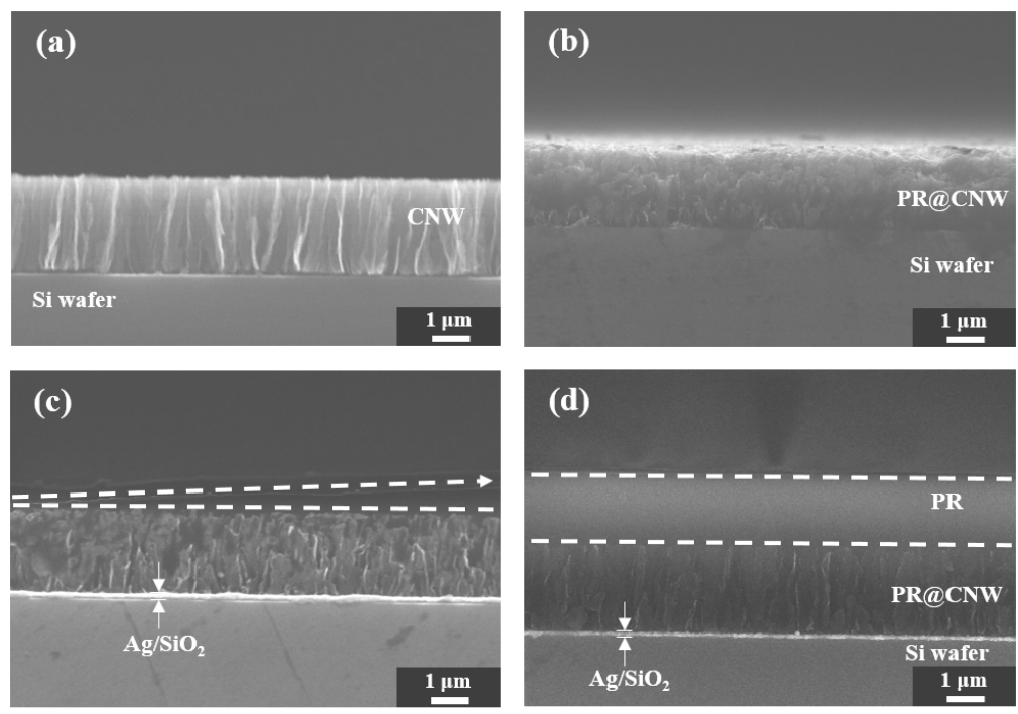

Figure 3. FESEM cross-sectional image of the (a) carbon nanowall (CNW), (b) photoresist (PR)@CNW, and (c) PR@CNW between the desired and non-removed areas, and the (d) PR-coated CNW. 
To determine the reason for the strong bond between the PR and the CNW, the contact angle between the DI water and the CNW was confirmed, as shown in Figure 4a. Water molecules are polar and do not mix well with non-polar molecules like oil. The contact angle of the CNW was $81^{\circ}$, which means that it was hydrophobic and non-polar. On the contrary, the SMCNW in Figure $4 \mathrm{~b}$ has a low contact angle with DI water due to activation of the functional group on the nanowall surface, and is hydrophilic and polar. The extreme change in the contact angle through surface modification of the nanowall can also be explained by the Laplace pressure, which results from the difference in surface energy between the nanowall and the buffer layer $[27,28]$. The PR that was used in this study was a non-polar molecule. As a result, the mixing of non-polar molecules caused strong adhesion, enabling the PR@CNW to remain even after the development process. Figure 4c explains why SMCNW has low adhesion to PR, unlike CNW. As with the polar molecular formation on the surface, which is the reason for carrying out the dehydration baking process, SMCNW was employed to reduce the adhesion between the CNW and the PR.

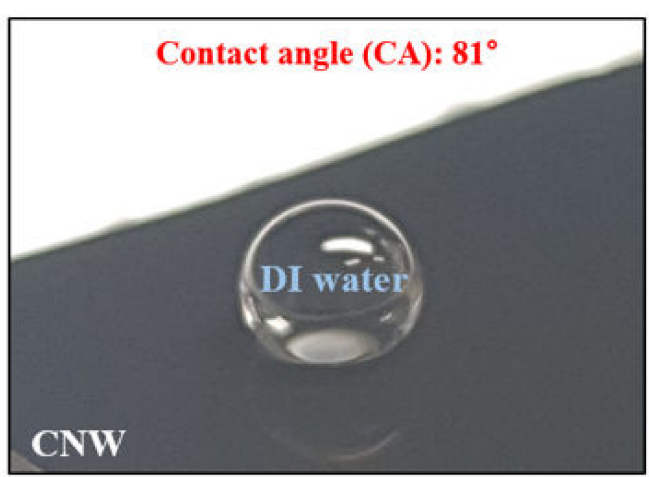

(a)

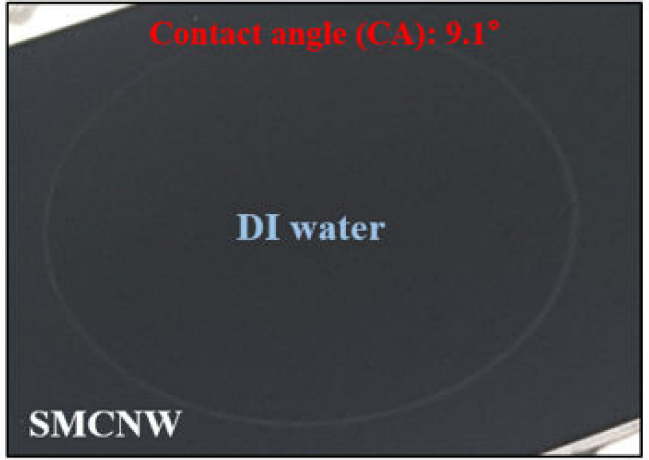

(b)

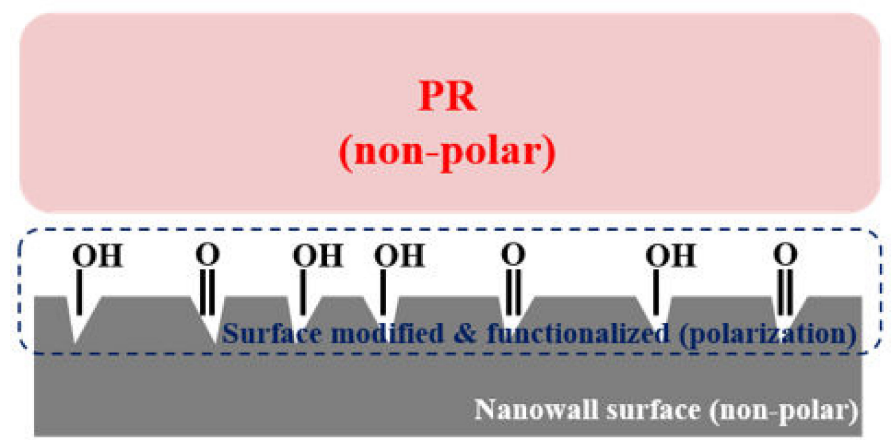

(c)

Figure 4. Contact angle of the (a) CNW and (b) surface-modified carbon nanowall (SMCNW). (c) Schematic of the positive PR-coated SMCNW.

An attempt was made to perform surface micromachining using the adhesion of the $\mathrm{Ag} / \mathrm{SiO} \mathrm{O}_{2}$ layer and CNW, without using a PR. Figure 5 shows a FESEM image after sonication of a CNW-grown sample in DI water without coating it with a PR. As the CNW grown directly on the Si wafer dissociated, the boundary with the $\mathrm{CNW}$ grown on the $\mathrm{Ag} / \mathrm{SiO}_{2}$ layer appeared as shown in Figure $5 \mathrm{a}$. The deformed $\mathrm{CNW}$ was observed after sonication, as shown in Figure 5b, to see if the boundary line was quite visible but had an effect on the CNW. As expected, some nanowalls had collapsed and were inappropriate for use with CNW surface micromachining that only employed adhesion. 


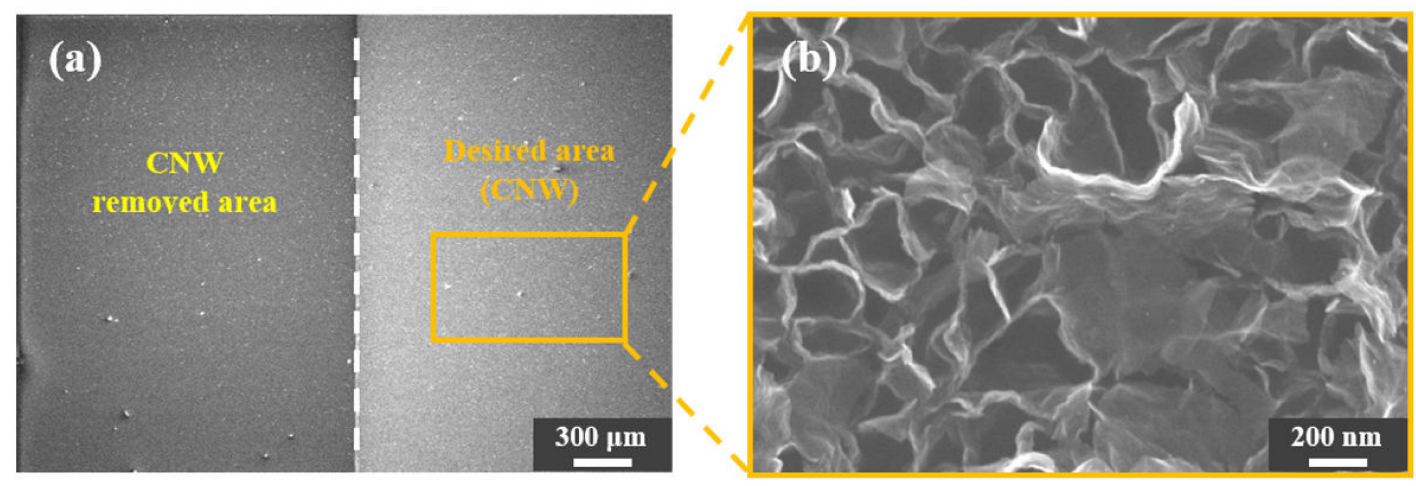

Figure 5. FESEM surface image (a) after sonication of the CNW grown on a Si wafer (removed area) and a $\mathrm{Ag} / \mathrm{SiO}_{2}$ layer (desired area) without PR coating. (b) FESEM image of $300 \mathrm{~K}$ magnification in the $\mathrm{CNW}$ grown on the desired area after sonication.

\subsection{Observation of the Variation in the SMCNW to which Surface Micromachining Technology Was Applied via Physical Force}

With regard to whether CNW is affected by a PR removal agent and a PR that has not been removed, PR cannot be applied to CNW in surface micromachining. Figure 6 shows whether the surface properties of SMCNW are changed by PR or not. Figure 6a shows that the SMCNW is removed through sonication, along with the boundary between the desired and removed areas. Figure $6 \mathrm{~b}$ shows that the SMCNW dissociated completely, with only $\mathrm{Si}$ atoms appearing. Comparing the CNW in Figure $6 \mathrm{c}$ and the SMCNW in Figure $6 \mathrm{~d}$ grown on the $\mathrm{Ag} / \mathrm{SiO}_{2}$ layer, it can be seen that the surface of the nanowall was roughened by surface modification. The functional group was attached to the roughened area for polarization to lower the adhesion to the PR, and the SMCNW obtained by lifting off the patterned PR from acetone is shown in Figure 6e. The SMCNW after PR removal did not show any noticeable changes from before PR removal, and the nanowall surface had the same rough appearance.

In CNW chemical bonding, there is $\mathrm{sp}^{3}$ bonding in the area where nanowalls are grown from the buffer layer, and since branches are grown from the nanowall, $\mathrm{sp}^{2}$ (a graphene layer of $\mathrm{CNW}$ ) and $\mathrm{sp}^{3}$ bonding exist simultaneously $[20,21]$. These two bonds can be seen on the CNW C1s peak in Figure 7a, and a small O1s peak caused by exposure to air appeared in the XPS survey. In the SMCNW XPS survey (Figure $7 \mathrm{~b}$ ), a Ag3d peak and an O1s peak larger than that of the CNW were identified. It can be inferred that the appearance of a Ag3d peak in the SMCNW XPS survey was due to the etching of the $\mathrm{O}_{2}$ plasma in the buffer layer. The O1s on the CNW surface had about 1.45 atomic \% while the $\mathrm{O} 1 \mathrm{~s}$ on the SMCW surface had about 14.3 atomic $\%$. Some shoulder and $\mathrm{C}-\mathrm{O}$ and $\mathrm{C}=\mathrm{O}$ peaks were identified in the C1s peak of the SMCNW, causing functional groups to form on the surface. Figure 7c shows the chemical properties of the SMCNW after the PR was removed, as obtained via XPS analysis. There were minimal differences in peak and even in components (O1s: 13.54 atomic \%), in Figure 7b, before PR coating. From some O1s component difference between SMCNW and PR-removed SMCNW, it can be inferred that the functional group on SMCNW surface is slightly modified by the remaining acetone solution after reacting with PR because of SMCNW solubility [29]. However, comparing the XPS analyses of their chemical bonding, the difference is meaningless. Therefore, the chemical properties of the PR-coated SMCNW and of the SMCNW from which the PR had been removed using acetone indicate that there were no changes in the chemical properties from before the PR coating. 

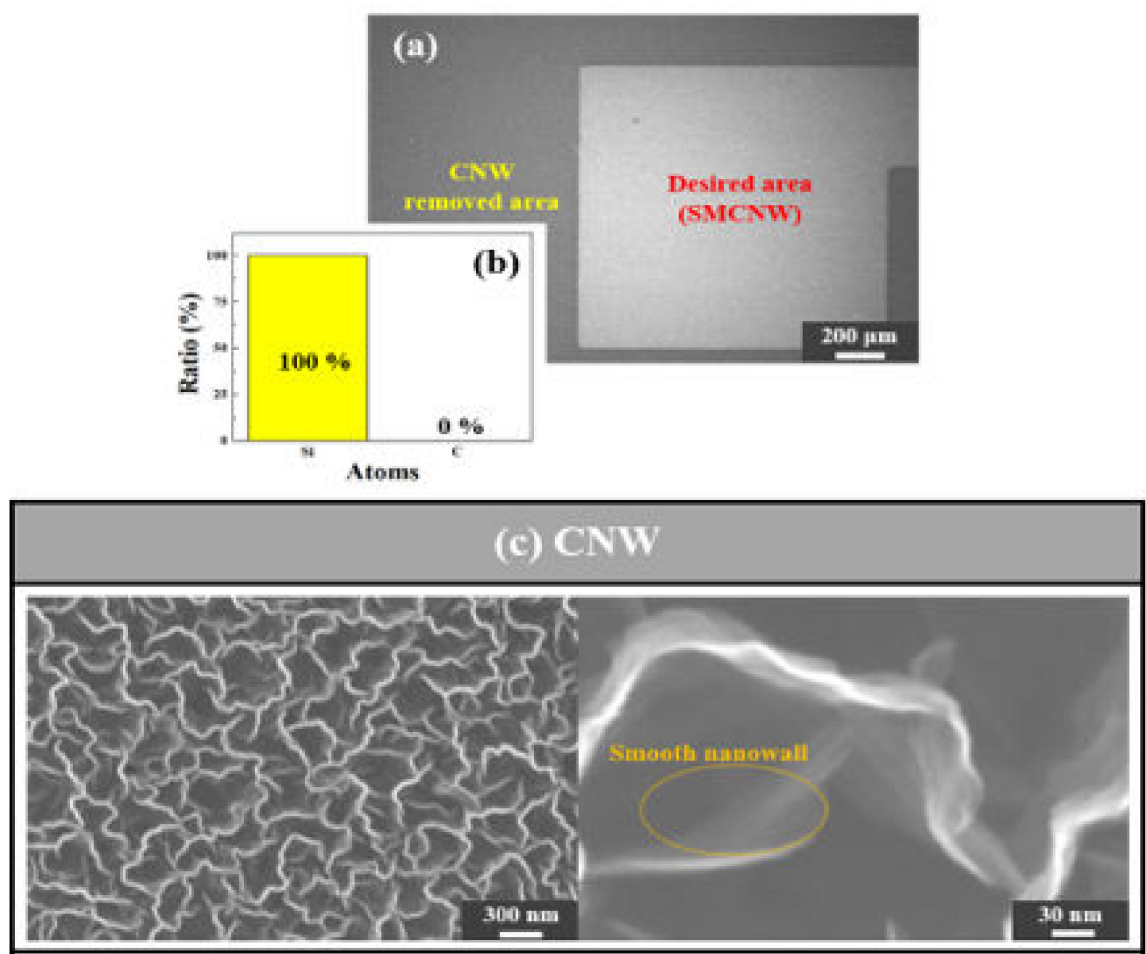

(d) SMCNW

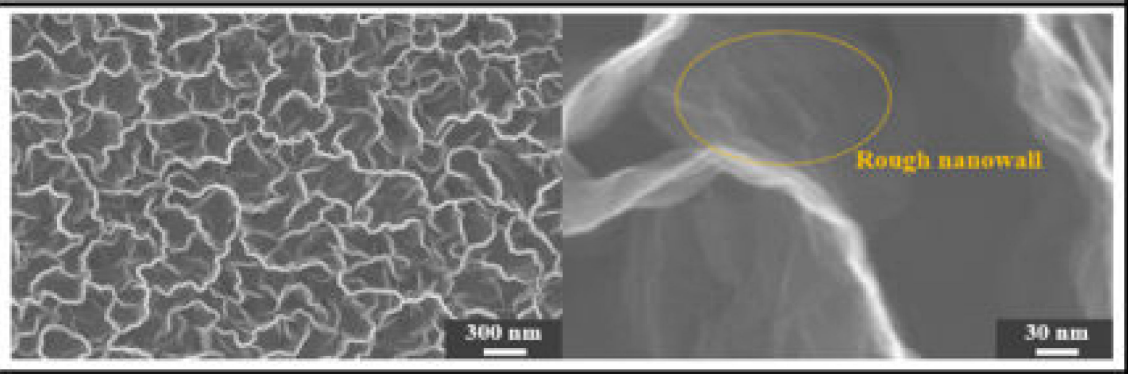

(c) SMCNW after PR lift off

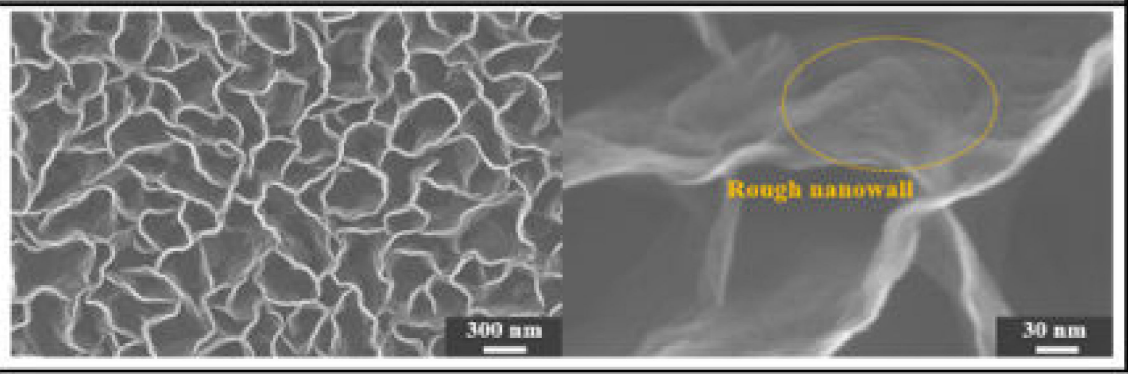

Figure 6. (a) FESEM surface image after applying the top-down surface micromachining technology to the SMCNW. (b) EDS analysis of the area where the CNW was removed through ultrasonic cleaning. FESEM surface image of the (c) CNW, (d) SMCNW, and (e) PR-removed SMCNW. 


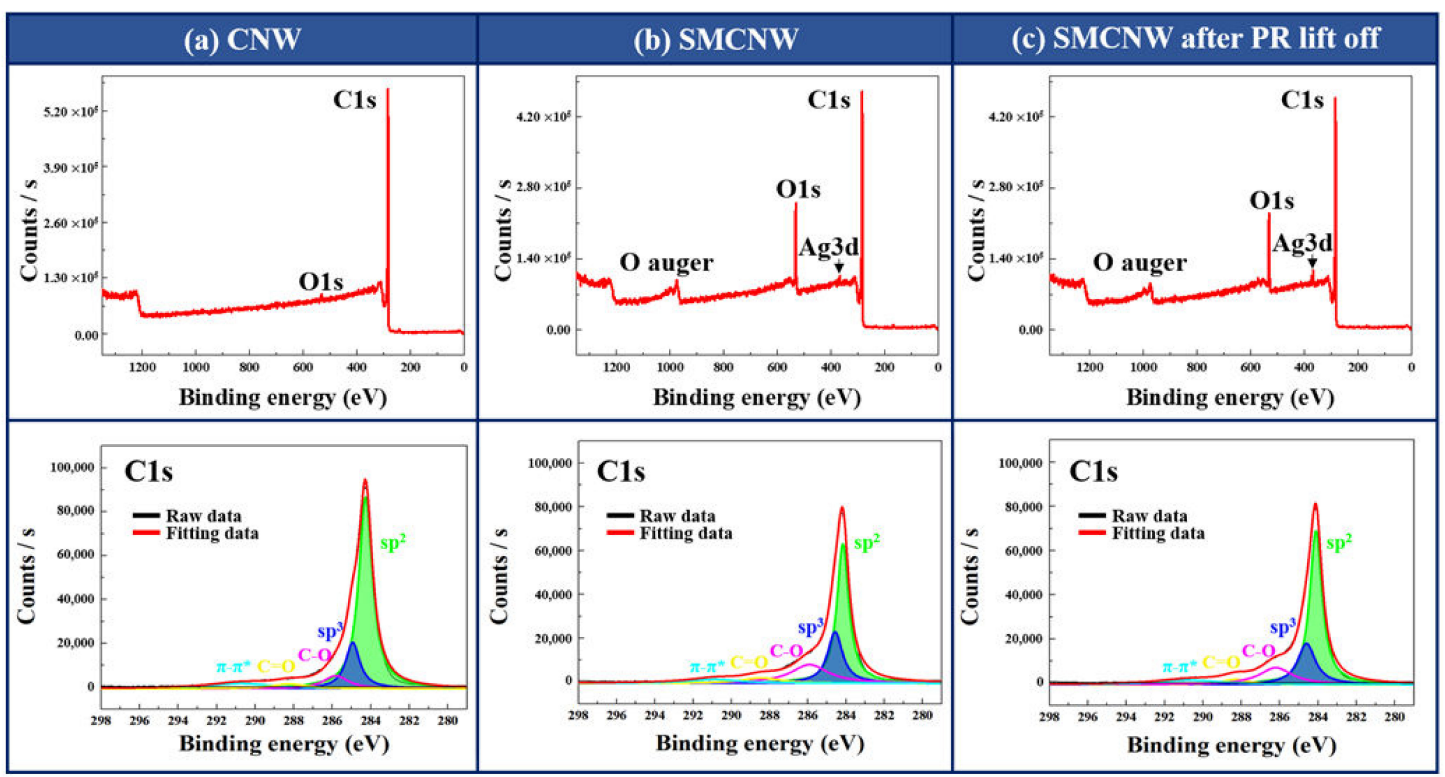

Figure 7. XPS analysis of the (a) CNW, (b) SMCNW, and (c) PR-removed SMCNW.

CNW has unique structural characteristics that differentiate it from other graphite-related materials. Figure 8 shows the Raman spectra of CNW, SMCNW, and PR-removed SMCNW, and the inherent peaks (i.e., D, D' (edge and defect of graphene), G (sp ${ }^{2}$ bonding), and 2D (thickness of graphene) peaks of graphite-related materials) are equally identified [29-33]. The results obtained through the five trials for each sample (CNW, SMCNW, and PR-removed SMCNW) showed almost constant intensity. The average $I_{D} / I_{G}$ (intensity of the D peak/intensity of the $G$ peak) of the SMCNW was smaller than that of the CNW. The bigger the $I_{D} / I_{G}$ ratio, the larger the defect. In the XPS analysis result shown in Figure 7, however, the SMCNW should have a higher $I_{D} / I_{G}$ ratio than the $C N W$ because the SMCNW was formed from some functional group that was released after the $\mathrm{sp}^{2}$ bonding. Nevertheless, the $I_{D} / I_{G}$ ratio of the SMCNW was smaller than that of the CNW. It can be inferred that the edge portion of the nanowall branches, which consisted of graphene layers, was reduced through $\mathrm{O}_{2}$ plasma etching. The number of graphene layers can be predicted from the $I_{2 D} / I_{G}$ (intensity of the 2D peak/intensity of the $G$ peak) ratio, and the larger the ratio is, the thinner the graphene layers. Therefore, the decrease in the $I_{2 D} / I_{G}$ ratio of the SMCNW can be attributed to the removal of the nanowall branches composed of relatively thin graphene layers compared to the nanowall. The structural properties of the SMCNW with the PR removed were almost identical to those of the SMCNW before PR coating. Therefore, it was demonstrated in this study that structural changes do not occur even after lifting off the PR from the SMCNW. 


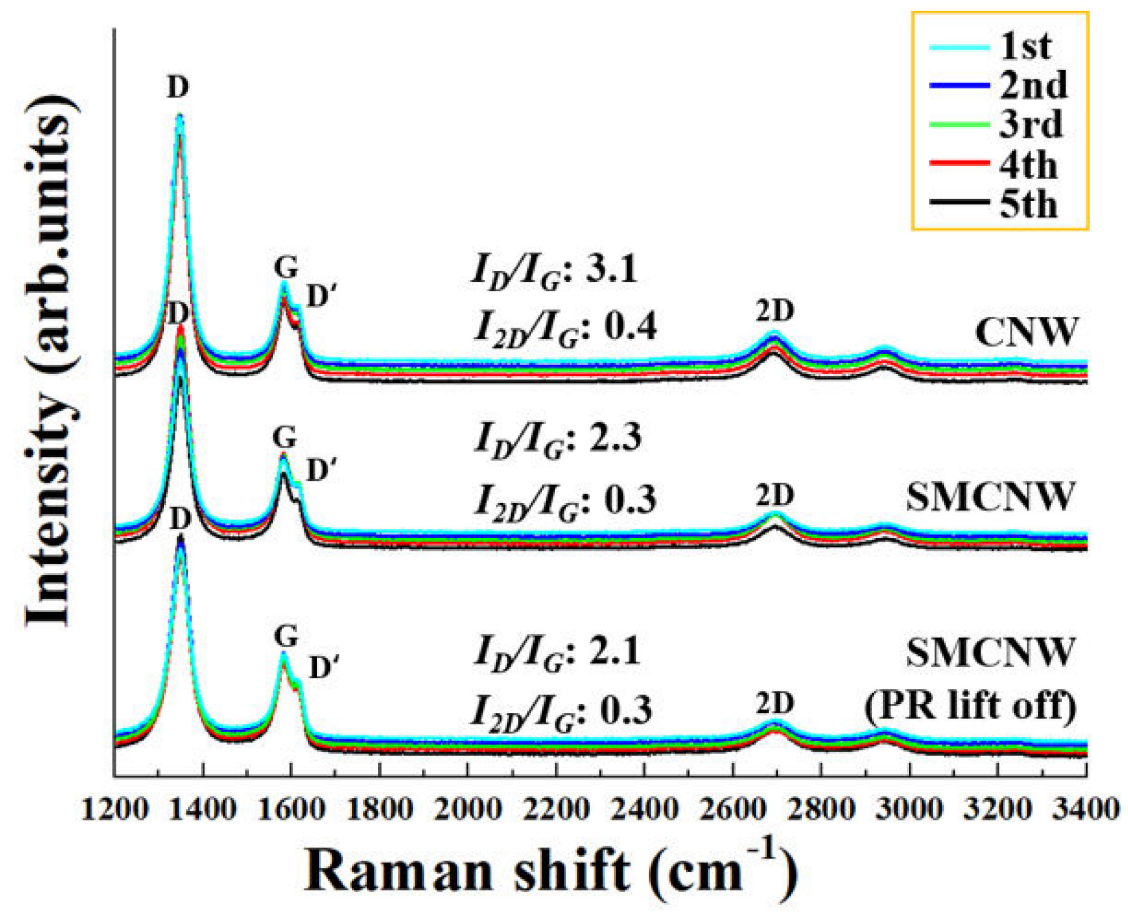

Figure 8. Raman spectra of the CNW, SMCNW, and PR-removed SMCNW.

\section{Conclusions}

Reported in this paper is a new carbon nanowall (CNW) top-down surface micromachining technology utilizing physical force. We propose a technology that grows directly on the substrate in a high temperature $\left(600{ }^{\circ} \mathrm{C}\right)$ rather than using an etchant or transcription. There are three key elements in this method. First, using the fact that CNW has low adhesion with $\mathrm{Si}$ wafer and is easily dissociated even when small physical forces are applied; an interlayer $\left(\mathrm{Ag} / \mathrm{SiO}_{2}\right)$ that enhances the adhesion between CNW and its under layer was deposited on the desired area. Second, since the non-polar PR and CNW are strongly bonded to each other, the CNW is surface-modified using oxygen plasma to attach functional groups and change its polarity. Third, in order to prevent the collapse of the surface-modified CNW (SMCNW) grown in the desired area due to the physical force, PR is coated and the unnecessary area is etched using sonication. SMCNW grown on $\mathrm{Si}$ wafer has low adhesion, so it can be easily removed by sonication and even more than half of it is dissolved along with UV light exposed PR in the developer. It was observed through energy-dispersive X-ray spectroscopy (EDS) analysis that the SMCNW etching site had been completely removed. In order to obtain the reliability of patterned SMCNW through this method, the surface, chemical, and structural properties of PR-removed SMCNW were investigated. All characteristics were not different from the pristine-SMCNW, indicating that surface micromachining was successful. The results obtained from this work can lead to the expansion of the CNW research field, which has not applied micromachining technology, and diversification of its application field.

Author Contributions: Conceptualization, H.C.; methodology, H.C.; writing-original draft, H.C.; writing-review and editing, S.K. and W.C.; visualization, S.K., S.L., Y.K., and J.H.K.; data curation, S.K., S.L., and Y.K.; resources, H.K.; formal analysis, J.H.K.; supervision, W.C. All authors have read and agreed to the published version of the manuscript.

Funding: This work was supported by the Korea Institute of Energy Technology Evaluation and Planning (KETEP), the Ministry of Trade, Industry, \& Energy (MOTIE) of the Republic of Korea (No. 20184030201900), and by a Korea Science and Engineering Foundation (KOSEF) grant funded by the South Korean government (MEST) (No. NRF-2018R1D1A1B07046804).

Conflicts of Interest: The authors declare no conflict of interest. 


\section{References}

1. Chang, T.-L.; Chou, C.-Y.; Wang, C.-P.; Teng, T.-C.; Han, H.-C. Picosecond laser-direct fabrication of graphene-based electrodes for a gas sensor module with wireless circuits. Microelectron. Eng. 2019, 210, 19-26. [CrossRef]

2. Wang, C.; Tong, H.; Lu, J.; Liu, B.; Zheng, F.; Tao, W.; Zhang, W.; Chen, Q. Boosting Oxygen Evolution Reaction on Graphene through Engineering Electronic Structure. Carbon 2020, 170, 414-420. [CrossRef]

3. Guo, S.; Zhang, D.; Zhou, J.; Deng, J.; Yu, Y.; Deng, J.; Cai, Q.; Li, Z.; Lu, W.; Chen, X. Enhanced infrared photoresponse induced by symmetry breaking in a hybrid structure of graphene and plasmonic nanocavities. Carbon 2020, 170, 49-58. [CrossRef]

4. Song, H.; Li, Q.; Zhang, Y. CNT-based sensor array for selective and steady detection of $\mathrm{SO}_{2}$ and $\mathrm{NO}_{\text {. }}$ Mater. Res. Bull. 2020, 124, 110772. [CrossRef]

5. Yaghoubi, A.; Ramazani, A. Anticancer DOX delivery system based on CNTs: Functionalization, targeting and novel technologies. J. Control. Release 2020, 327, 198-224. [CrossRef]

6. Liu, Y.; He, D.; Dubrunfaut, O.; Zhang, A.; Zhang, H.; Pichon, L.; Bai, J. GO-CNTs hybrids reinforced epoxy composites with porous structure as microwave absorbers. Compos. Sci. Technol. 2020, 200, 108450. [CrossRef]

7. Dong, Y.; Fan, X.; Wei, H.; Hou, Z.; Li, M.; Qu, Q.; Yin, X.; Cheng, L.; Zhang, L. A lightweight $\mathrm{CNWs}-\mathrm{SiO}_{2} / 3 \mathrm{Al}_{2} \mathrm{O}_{3} \cdot 2 \mathrm{SiO}_{2}$ porous ceramic with excellent microwave absorption and thermal insulation properties. Ceram. Int. 2020, 46, 20395-20403. [CrossRef]

8. Ren, F.; Xue, J.; Liu, X.; Cheng, L. In situ construction of CNWs/SiC-NWs hybrid network reinforced SiCN with excellent electromagnetic wave absorption properties in X band. Carbon 2020, 168, 278-289. [CrossRef]

9. Shin, J.H.; Park, H.J.; Song, Y.I.; Choi, Y.S.; Suh, S.-J. Morphological optimization and nitrogen functionalization of vertically oriented CNW for high performance electrical double layer capacitor electrode. Electrochim. Acta 2020, 348, 136210. [CrossRef]

10. Masoudzadeh, F.; Jamshidi, M.; Fasihi, M. Preparation and application of cellulose nano whiskers (CNWs) in engineered cementitious composites. J. Build. Eng. 2019, 21, 213-221. [CrossRef]

11. Makris, T.D.; Giorgi, L.; Giorgi, R.; Lisi, N.; Salernitano, E. CNT growth on alumina supported nickel catalyst by thermal CVD. Diam. Relat. Mater. 2005, 14, 815-819. [CrossRef]

12. Gnanaprakasa, T.J.; Gu, Y.; Eddy, S.K.; Han, Z.; Beck, W.J.; Muralidharan, K.; Raghavan, S. The role of copper pretreatment on the morphology of graphene grown by chemical vapor deposition. Microelectron. Eng. 2015, 131, 1-7. [CrossRef]

13. Naghdi, S.; Rhee, K.Y.; Park, S.-J. A catalytic, catalyst-free, and roll-to-roll production of graphene via chemical vapor deposition: Low temperature growth. Carbon 2018, 127, 1-12. [CrossRef]

14. Cushing, G.W.; Johánek, V.; Navin, J.K.; Harrison, I. Graphene Growth on Pt(111) by Ethylene Chemical Vapor Deposition at Surface Temperatures near 1000 K. J. Phys. Chem. C 2015, 119, 4759-4768. [CrossRef]

15. Vizireanu, S.; Stoica, S.D.; Luculescu, C.; Nistor, L.C.; Mitu, B.; Dinescu, G. Plasma techniques for nanostructured carbon materials synthesis. A case study: Carbon nanowall growth by low pressure expanding RF plasma. Plasma Sources Sci. Technol. 2010, 19, 34016. [CrossRef]

16. Lisi, N.; Giorgi, R.; Re, M.; Dikonimos, T.; Giorgi, L.; Salernitano, E.; Gagliardi, S.; Tatti, F. Carbon nanowall growth on carbon paper by hot filament chemical vapour deposition and its microstructure. Carbon 2011, 49, 2134-2140. [CrossRef]

17. Dikonimos, T.; Giorgi, L.; Giorgi, R.; Lisi, N.; Salernitano, E.; Rossi, R. DC plasma enhanced growth of oriented carbon nanowall films by HFCVD. Diam. Relat. Mater. 2007, 16, 1240-1243. [CrossRef]

18. Hiramatsu, M.; Shiji, K.; Amano, H.; Hori, M. Fabrication of vertically aligned carbon nanowalls using capacitively coupled plasma-enhanced chemical vapor deposition assisted by hydrogen radical injection. Appl. Phys. Lett. 2004, 84, 4708-4710. [CrossRef]

19. Shih, W.-C.; Jeng, J.-M.; Huang, C.-T.; Lo, J.-T. Fabrication of carbon nanoflakes by RF sputtering for field emission applications. Vacuum 2010, 84, 1452-1456. [CrossRef]

20. Zhu, M.; Wang, J.; Holloway, B.C.; Outlaw, R.; Zhao, X.; Hou, K.; Shutthanandan, V.; Manos, D.M. A mechanism for carbon nanosheet formation. Carbon 2007, 45, 2229-2234. [CrossRef]

21. Zhao, J.; Shaygan, M.; Eckert, J.; Meyyappan, M.; Rümmeli, M.H. A Growth Mechanism for Free-Standing Vertical Graphene. Nano Lett. 2014, 14, 3064-3071. [CrossRef] [PubMed] 
22. Mironovich, K.V.; Itkis, D.M.; Semenenko, D.A.; Dagesian, S.A.; Yashina, L.V.; Kataev, E.Y.; Mankelevich, Y.A.; Suetin, N.V.; Krivchenko, V.A. Tailoring of the carbon nanowall microstructure by sharp variation of plasma radical composition. Phys. Chem. Chem. Phys. 2014, 16, 25621-25627. [CrossRef] [PubMed]

23. Cho, H.J.; Kondo, H.; Ishikawa, K.; Sekine, M.; Hiramatsu, M.; Hori, M. Density control of carbon nanowalls grown by $\mathrm{CH}_{4} / \mathrm{H}_{2}$ plasma and their electrical properties. Carbon 2014, 68, 380-388. [CrossRef]

24. Zang, X.; Zhou, Q.; Chang, J.; Liu, Y.; Lin, L. Graphene and carbon nanotube (CNT) in MEMS/NEMS applications. Microelectron. Eng. 2015, 132, 192-206. [CrossRef]

25. Vesel, A.; Mozetič, M.; Panjan, P.; Hauptman, N.; Klanjsek-Gunde, M.; Balat-Pichelin, M. Etching of carbon-tungsten composite with oxygen plasma. Surf. Coat. Technol. 2010, 204, 1503-1508. [CrossRef]

26. Pears, K.A.; Stolze, J. Carbon etching with a high density plasma etcher. Microelectron. Eng. 2005, 81, 7-14. [CrossRef]

27. Choi, H.; Kwon, S.H.; Kang, H.; Kim, J.H.; Choi, W. Analysis of plasma-grown carbon oxide and reduced-carbon-oxide nanowalls. RSC Adv. 2020, 10, 9761-9767. [CrossRef]

28. Zhang, L.; Sun, Z.; Qi, J.; Shi, J.; Hao, T.; Feng, J. Understanding the growth mechanism of vertically aligned graphene and control of its wettability. Carbon 2016, 103, 339-345. [CrossRef]

29. Kwon, S.; Choi, H.; Lee, S.; Kim, Y.; Choi, W.; Kang, H. Solubility of modified catalyst-free carbon nanowall with organic solvents. Appl. Surf. Sci. 2020, 529, 147161. [CrossRef]

30. Malard, L.M.; Pimenta, M.A.; Dresselhaus, G.; Dresselhaus, M.S. Raman spectroscopy in graphene. Phys. Rep. 2009, 473, 51-87. [CrossRef]

31. Graf, D.; Molitor, F.; Ensslin, K.; Stampfer, C.; Jungen, A.; Hierold, C.; Wirtz, L. Spatially Resolved Raman Spectroscopy of Single- and Few-Layer Graphene. Nano Lett. 2007, 7, 238-242. [CrossRef] [PubMed]

32. Ferrari, A.; Meyer, J.C.; Scardaci, V.; Casiraghi, C.; Lazzeri, M.; Mauri, F.; Piscanec, S.; Jiang, D.; Novoselov, K.S.; Roth, S.; et al. Raman Spectrum of Graphene and Graphene Layers. Phys. Rev. Lett. 2006, 97, 187401. [CrossRef] [PubMed]

33. Soin, N.; Roy, S.S.; Ray, S.C.; McLaughlin, J. Excitation energy dependence of Raman bands in multiwalled carbon nanotubes. J. Raman Spectrosc. 2010, 41, 1227-1233. [CrossRef]

(C) 2020 by the authors. Licensee MDPI, Basel, Switzerland. This article is an open access article distributed under the terms and conditions of the Creative Commons Attribution (CC BY) license (http://creativecommons.org/licenses/by/4.0/). 